\title{
Spin-Orbital Effect of Diexciton in Quantum Dots
}

\author{
Are zu Jahanshir ${ }^{1,2}$ \\ ${ }^{1}$ Sci. \& Tech. Park, University of Tehran, Tehran, Islamic Republic of Iran \\ ${ }^{2}$ Faculty of physics, department of theoretical physics, FKN University, Almaty, 92 Tolebi St. Kazakhstan
}

\begin{abstract}
Great efforts have been made to understand how bound state arise in the formalis m of quantum field theory and to work out effective methods to calculate all characteristics of these bound states, especially their masses and binding energy. The analysis of a bound state is simplest when constituent particles can be considered to be nonrelativistic, i.e. when they travel at speeds considerably less than " $c$ ". We studied an exotic system (state) on the basis of investigation of asymptotic behavior of the loop function for the scalar partic les in the external gauge field and determined it for the scalar particles in the with relativistic feature of interaction and have been able to obtain the mass spectrum of bound state, the constituent mass of diexciton and spin interactions and find out that determining the mass of the bound state systems requires; first of all, determin ing the Eigenvalue of the Hamiltonian with Coulo mb potential and then calculating the mass and binding energy or spin interactions of diexciton system which we could achieve. The current paper has calculated energy spectrum and constituents mass of particles in limited core mass conditions and we could conclude that constituent mass of particle in the system is different from mass in free states. So having below conditions form inhomogeneous multi-layer environment in nanostructure $\left(A_{3} B_{5} \mid A_{x} C_{x-1} B_{5}\right)$ in mind, and using oscillator representation method, spin Hamiltonian coefficient of die xciton system with effective confinement potential, Coulo mb effect between particles, and spin effect, will be discussed. Relation between distances properties of diexciton system and distance between two electrons in quantum dots and dependency relation will be determined in conclusion.
\end{abstract}

Keywords Exciton System, Oscillator Representation Method, Spin-Orb ital and Spin-Spin Interactions, Effective Mass, Constituent Mass

\section{Introduction}

Electrons and holes in microscopic semiconductor structures can display astounding quantum behavior. These structures, namely exciton are expected to be the bas is of new generation of electronic and optoelectronic and nano-structures [1,2]. There fore description of the bound states of electron and hole is one of the general problems in quantum mechanics and nano quantum dots. This problem is studied by many authors and it is well known but in this article we try to present new approaches which help us to describe and determine better and more useful. One of the corrections in particle physics can be classified as relativ istic.

At present the technical achievements make it possible to create the exotic state with electron and exciton so in this quantum- relativistic state, the calculation of relativistic corrections becomes necessary. However, the conventional prescription of the calculation of the relativistic nature interaction within the framework of the phenomenological potential model of is absent at the moment and the theoretical

* Corresponding author:

aresuj@gmail.com (Arezu Jahanshir)

Published online at http://journal.sapub.org/ajcmp

Copyright (C) 2012 Scientific \& Academic Publishing. All Rights Reserved models intended for describing the relativistic corrections to the spectrum are limited to the lowest order on the coupling constant. The other powerful stimulus for further development of the bound state theory is provided by the spectacular experimental progress in precise measurements of atomic energy levels. Calculation and analysis of energy spectrum in Coulo mb potential of electron and exciton in relativistic conditions due to requirement of using higher grades of relativistic corrections have attracted physics theoreticians.

We will consider this problem, according to the asymptotic behavior of the loop function in the scalar electrodynamics field and method oscillator representation in quantum physics [3]. The method presented in this paper considers relativistic effect that it concepts energy spectrum, mass and constituent mass in the exotic system and analytical is calculated with Coulomb interaction. This work is devoted to study the bound states problems, which will be carried out on the research basis asymptotical behavior of two scalar particles in external gauge field. In this case, loop function of two scalar particles with different masses $m_{1}$ and $m_{2}$, with average on external statistical field is considered and the Green function is used. The polarization operator in an external electromagnetic field looks like[4]:

$$
\Pi(x-y)=\left\langle G_{m_{1}}(x, y \mid A) G_{m_{2}}^{*}(y, x \mid A)\right\rangle_{A}
$$


The Green function $G(x, y \mid A)$ for scalar particles in an external field is determined fro $m$ the equation:

$$
\left[\left(i \frac{\partial}{\partial x_{\alpha}}+\frac{\alpha}{c \hbar} A_{\alpha}(x)\right)^{2}+\frac{c^{2} m^{2}}{\hbar^{2}}\right] G(x, y \mid A)=\delta(x-y)
$$

Where $m$ is the mass of a scalar particle, and $\alpha$ is the coupling constant of interaction. The gauge field averaging defines as follows:

$$
\begin{gathered}
\left\langle\exp \left\{i \int d x A_{\alpha}(x) J_{\alpha}(x)\right\}\right\rangle_{A}= \\
=\exp \left\{-\frac{1}{2} \iint d x_{1} d x_{2} J_{\alpha}(x) D_{\alpha \beta}(x-y) J_{\beta}(y)\right\}
\end{gathered}
$$

Here $J_{\alpha}(x)$ is a real current, and

$$
D_{\mu \nu}(x-y)=\delta_{\mu \nu} D(x-y)+\partial_{\eta \nu}^{2} \tilde{D}(x-y)
$$

By using the form of the functional integral we can represent green function as follow [5]:

$$
\begin{aligned}
& G(x, y \mid A)=\int_{0}^{\infty} \frac{d s}{(4 s \pi)^{2}} \exp \left\{-s m^{2}-\frac{(x-y)^{2}}{4 s}\right\} \times \\
& \times \int d \sigma_{\beta} \exp \left\{\operatorname{ig} \int_{0}^{1} d \xi \frac{\partial Z_{\alpha}(\xi)}{\partial \xi} A_{\alpha}(\xi)\right\}= \\
& \int_{0}^{\infty} \frac{1}{(4 \pi s)^{2}} \exp \left(s m^{2}-\frac{(x-y)^{2}}{4 s}\right) d s \int d \vec{\gamma}_{\mu} \times \\
& \times e^{-\left(\int d \beta \vec{\gamma}_{\mu}^{2}-4 \alpha g^{2} \int_{0}^{s} \int_{0}^{s} d \beta_{1} d \beta_{2} \vec{\gamma}_{\mu}\left(\beta_{1}\right) \vec{\gamma}_{\nu}\left(\beta_{2}\right) D_{\mu \nu}\left(Z\left(\beta^{\prime}\right)\right)\right.}
\end{aligned}
$$

where used the notations

$$
\begin{aligned}
& Z_{\alpha}(\xi)=(x-y)_{\alpha}+y_{\alpha} \xi-2 \sqrt{s} B_{\alpha}(\xi) \\
& d \sigma_{\beta}=N \delta \vec{B} \exp \left\{-1 / 2 \int_{0}^{1} d \xi \dot{\vec{B}}^{2}(\xi)\right\}
\end{aligned}
$$

with the conditions:

$$
B_{\alpha}(0)=B_{\alpha}(1) ; \quad \int d \sigma_{\beta}=1
$$

The mass of the bound state in the extreme limit is determined by the equation[4,5]:

$$
\left.M=-\lim _{|x-y| \rightarrow \infty} \frac{\ln \Pi(x-y)}{|x-y|}\right)
$$

After averaging over the field $A_{\alpha}(x)$ we have (for details of an evaluation see[5]):

here:

$$
\begin{gathered}
\Pi(x-y)=\int_{0}^{\infty} \int_{0}^{\infty} \frac{d u_{1} d u_{2}}{\left(8 x \pi^{2}\right)^{2}} \cdot J_{\mu}\left(\mu_{1}, \mu_{2}\right) \times \\
\times \exp \left\{-\frac{x}{2}\left(\frac{m_{1}^{2}}{\mu_{1}}+\mu_{1}\right)-\frac{x}{2}\left(\frac{m_{2}^{2}}{\mu_{2}}+\mu_{2}\right)\right\} \\
J\left(\mu_{1}, \mu_{2}\right)=A \iint \delta \vec{r}_{1} \delta \vec{r}_{2} \times \\
\times \exp \left\{-\frac{1}{2} \int_{0}^{1 x} d \tau\left(\mu_{1} \dot{\vec{r}}^{2}{ }_{1}(\tau)+\mu_{2} \dot{\vec{r}}^{2}{ }_{2}(\tau)\right)\right\} \exp \left\{-W_{i, j}\right\}
\end{gathered}
$$

Functional integral in (8) is similar to the Feynman Path integral trajectories [5] in nonrelativistic quantum mechanics [9] for the motion of two particles with masses $\mu_{1}$ and $\mu_{2}$. The interaction of these particles is described by the nonlocal functional $W_{i j}$, in which are contained both potential and non-potential interaction (see appendix 1). Taking into account (8) and (9) in the extreme limit from (7) for the mass of the bound state we get (for detail see[5-7]):

$$
\begin{gathered}
M=\sqrt{m_{1}^{2}-2 \mu^{2} \frac{\partial E(\mu)}{\partial \mu}}+\sqrt{m_{2}^{2}-2 \mu^{2} \frac{\partial E(\mu)}{\partial \mu}}+ \\
+\mu \frac{\partial E(\mu)}{\partial \mu}+E(\mu)
\end{gathered}
$$

where the parameter $\mu$ is determined from the equation:

$$
\begin{gathered}
\frac{1}{\mu}=\frac{1}{\mu_{1}}+\frac{1}{\mu_{2}}= \\
\frac{1}{\sqrt{m_{1}^{2}-2 \mu^{2} E^{\prime}(\mu)}}+\frac{1}{\sqrt{m_{2}^{2}-2 \mu^{2} E^{\prime}(\mu)}}
\end{gathered}
$$

and $E(\mu)$ - is the eigenvalue of the nonrelativistic Hamiltonian. Parameters $\mu_{1}$ and $\mu_{2}$ are mass components of the bound state (constituent mass of particles), which are different from the masses $m_{1}, m_{2}$ of free condition and also can be found with the help of conditions:

$$
\lim _{|x| \rightarrow \infty} J\left(\mu_{1}, \mu_{2}\right)=\exp \left\{-x E\left(\mu_{1}, \mu_{2}\right)\right\}
$$

\section{Exciton's Hamiltonian in Relativistic Corrections}

As we know one of the basic problems of nonrelativistic quantum mechanics is to determine the energy spectrum and eigenvalue of the nonrelativistic Hamiltonian of system described by the Schrödinger equation with a special potential. Complete solution of this equation has been found in 1965 (for a detailed historical literature see[8] and Landau 1977[9], for the Coulomb potential. The oscillator representation method implies that a wave function, being a bound ground state of quantum system with a good looking potential, is expanded over the oscillator basis in which coordinate and momentum are expressed through the creation and annihilation operators.

The oscillator representation method in the nonrelativistic Schrödinger equation is proposed to calculate the energy spectrum for central symmetric potential allowing the existence of bound state. (for details see[10]). It is well known that for determin ing the mass of the bound state systems first of all one should determine the eigenvalue of the Hamiltonian with Coulomb potential. For this aim we consider the following Schrödinger equation[11,12]:

$$
\frac{1}{2 \mu} \vec{P}^{2}+\frac{z \alpha}{r} \Psi=E(\mu) \Psi
$$

$\alpha=e^{2} / 4 \pi$ - is the coupling constant of electromagnetic interaction. $z$-electron and hole charge number.

This equation for the wave function of orbital excitation $\ell^{2}=\ell(\ell+1)$ is: 


$$
\left\{-\frac{1}{2 \mu} \cdot\left(\frac{\partial^{2}}{\partial r^{2}}+\frac{2}{r} \frac{\partial}{\partial r}-\frac{\hat{\ell}}{r^{2}}\right)-\frac{z \alpha}{r}\right\} \Psi=E(\mu) \Psi(14)
$$

and at large distance, for the Coulomb potential the as ymptotic behavior of wave function is well known[12,13].

We considered this equation as the condition for the determination of the energy spectrum of the in itial system. By using oscillator representation method and techniques of quantum filed theories represent the canonical variables in terms of the creation $\left(a^{+}\right)$and annihilation $\left(a^{-}\right)$operators in the $R^{d}$ space:

$$
\begin{gathered}
q_{i}=\frac{a_{j}+a_{j}^{+}}{\sqrt{2 \omega}}, \\
p_{j}=\sqrt{\frac{\omega}{2}} \cdot \frac{a_{j}-a_{j}^{+}}{i}, \\
{\left[a_{j}, a_{j}^{+}\right]=\delta_{i, j} \quad j=1,2, \ldots d .}
\end{gathered}
$$

here $\omega$ is the oscillator frequency which has been known as Hamiltonian of free oscillator and can write as follow (for more detail see[11]):

$$
H_{0}=\omega\left(a_{j}^{+} a_{j}\right)
$$

We have to modify the variables in the starting Schrödinger equation. In this case, we used the substitution $r=q^{2 \rho}$, and found the following conditions [14,15]:

$$
\begin{gathered}
\frac{\partial^{2}}{\partial r^{2}}=\frac{q^{2-4 \rho}}{4 \rho^{2}}\left(\frac{\partial^{2}}{\partial q^{2}}+\frac{1-2 \rho}{q} \frac{\partial}{\partial q}\right) \\
\frac{\partial}{\partial r}=\frac{q^{1-2 \rho}}{2 \rho} \frac{\partial}{\partial q} \\
\frac{\partial^{2}}{\partial r^{2}}+\frac{2}{r} \frac{\partial}{\partial r}=\frac{q^{2-4 \rho}}{4 \rho^{2}}\left(\frac{\partial^{2}}{\partial q^{2}}+\frac{1-2 \rho}{q} \frac{\partial}{\partial q}\right)
\end{gathered}
$$

After simple calcu lations we obtain Schrödinger equation in os cillator representation method (for the Coulo mb potential $\rho=1$ ):

$$
\begin{gathered}
E(\mu) \Psi= \\
\left(-\frac{1}{2 \mu}\left(\frac{q^{2}}{4}\left(\frac{\partial^{2}}{\partial q^{2}}-\frac{1}{q} \frac{\partial}{\partial q}\right)-\frac{\ell^{2}}{q^{2}}\right)-\frac{z \alpha}{r}\right) \Psi
\end{gathered}
$$

Now we try to describe interactions in nano- quantum dots. Energy is absorbed by electrons presented in semiconductor, which means as activating electron from capacity band to conductive ban. Electron moves to conductive band and establish a connection with its position in capacity band (hole: hole with positive charge) under Coulomb potential gravity.

Electron and the hole start to spin around themselves and create an exciton system. Exciton system is relatively a stable structure, which in this field is considered as a long time life. Interaction and mutual effect of each layer with other layer in inhomogeneous environment is explained through electrostatic forces, electron relative potential (resulted from dissemination of charge and electron flow, which result to formation of related electromagnetic field between electrons), spin effects and particles' complementary effects[16].

In an effective mass approximation the Hamiltonian of exciton is $(\hbar=c=1)$ :

$$
H=-\frac{1}{2 m_{e}} \nabla_{r_{e}}^{2}-\frac{1}{2 m_{h}} \nabla_{r_{e}}^{2}-\frac{z^{2}}{\varepsilon\left|r_{e}-r_{h}\right|}
$$

$m_{e}, m_{h}$-effective mass of electron and hole. Making the co-ordinate transformation ${ }^{1}$ the Hamiltonian may be written[17]:

$$
H=-\frac{1}{2 \mu} \nabla_{r}^{2}-\frac{z^{2}}{\varepsilon \cdot r}
$$

$\mu$ - is the reduce mass.

Hamiltonian for diexciton as we now the complete Schrödinger equation for diexciton so rewrite[18]:

$$
\begin{aligned}
& H=H_{0}+\hat{H}_{\text {nonpert. }}=\hat{H}_{\text {spin }}+\Delta \hat{H}= \\
& \frac{1}{2} \sum_{i=1}^{n} m_{i} v_{i}^{2}+\sum_{i=1}^{n-1} \sum_{j=}^{n} \\
& i<j \\
& 2 \frac{1}{k} \frac{e_{i} e_{j}}{\left|r_{i}-r_{j}\right|}++\hat{H}_{\text {spin }}+\Delta \hat{H}
\end{aligned}
$$

Electrostatic force on assumed positive charges (visual charges) in distance of $h$ from the border of two environments with different dielectric coefficient will be as follows [19]:

$$
F(h)=\frac{z z^{\prime} \cdot e^{2}}{4 \pi \varepsilon \varepsilon_{0}(2 h)^{2}} \cdot \frac{\varepsilon^{\prime}-\varepsilon}{\varepsilon^{\prime}+\varepsilon}
$$

Therefore, this assumed positive charge (visual charge) on the border of assumed inho mogeneous system will be:

$$
\begin{gathered}
z_{1}=\frac{\varepsilon_{1}-\varepsilon}{\varepsilon_{1}+\varepsilon} e^{+}, \quad z_{2}=\frac{\varepsilon_{2}-\varepsilon}{\varepsilon_{2}+\varepsilon} e^{+} \\
\varepsilon_{1}, \varepsilon_{2}>\varepsilon
\end{gathered}
$$

\section{Four-particle Spin Interaction in Quantum Dots}

As we know all Hamilton of particles should be determined through surveying spin-spin and spin-orbital effect between particles in quantum dots. So final potential in quantum dots will be equal to vector potential resulted from uni-photon transaction and the potential resulted from electrons' confinement and spin effect: (complementary Hamilton's effect $(\Delta \hat{H})$ and relative potential $\left(U_{s}(x)\right)$ have been omitted due to simplicity):

\footnotetext{
1 Jacobian transformation.
} 


$$
\begin{aligned}
& U(x)=U_{v}(x)+U_{S}(x)+\hat{H}_{\text {spin }}, \\
& \hat{H}_{\text {spin }}=\hat{H}_{L S}+\hat{H}_{S S}
\end{aligned}
$$

Hamiltonian of spin-orb ital interaction is:

$$
\begin{aligned}
& H_{L S}= \\
& \left(\begin{array}{l}
\left.\frac{1}{4 r}\left(\frac{1}{\mu_{1}^{2}}+\frac{1}{\mu_{2}^{2}}+\frac{4}{\mu_{1} \mu_{2}}\right)+\right) \\
\frac{1}{4 r}\left(\frac{1}{\mu_{1}^{2}}-\frac{1}{\mu_{2}^{2}}\right)\left(\vec{L} \vec{S}_{-}\right)
\end{array}\right) \frac{\partial V_{v}(r)}{\partial r}- \\
& -\left(\begin{array}{l}
\left.\frac{1}{4 r}\left(\frac{1}{\mu_{1}^{2}}+\frac{1}{\mu_{2}^{2}}\right)\left(\vec{L} \vec{S}_{+}\right)+\right) \\
\frac{1}{4 r}\left(\frac{1}{\mu_{1}^{2}}-\frac{1}{\mu_{2}^{2}}\right)\left(\vec{L} \vec{S}_{-}\right)
\end{array}\right) \frac{\partial V_{S}(r)}{\partial r}
\end{aligned}
$$

Hamiltonian of spin-spin interaction is:

$$
H_{S S}=\frac{1}{12 \mu_{1} \mu_{2}} \cdot\left(\vec{S}_{1} \vec{S}_{2}\right) \cdot \Delta V_{v}(r)
$$

where

$$
\begin{aligned}
& \vec{S}_{ \pm}=\vec{S}_{1} \pm \vec{S}, \\
& \vec{S}_{12}=\frac{4}{(2 \ell+3)(2 \ell-1)} \times \\
& \times\left(\vec{L}^{2} \cdot \vec{S}^{2}-\frac{3}{2}(\vec{L} \vec{S})-3(\vec{L} \vec{S})^{2}\right)
\end{aligned}
$$

$S_{i^{-}}$spin of particles and we can determine by table 1 .

Table 1. Spin-orbital and spin-spin ,matrix elements.

\begin{tabular}{|c|c|c|c|}
\hline & $\mathrm{S}=2$ & $\mathrm{~S}=0$ & $\mathrm{~S}=-2$ \\
\hline$(\vec{L} \vec{S})$ & $2 \ell$ & 0 & $-2 \ell$ \\
\hline$\left(\vec{S}_{1} \vec{S}_{2}\right)$ & 1 & -2 & -1 \\
\hline$S_{12}$ & $-\frac{3 \cdot 4 \cdot \ell}{2 \ell+3}$ & 0 & $-\frac{3 \cdot 4 \cdot \ell}{2 \ell+3}$ \\
\hline
\end{tabular}

Election of distance between to Fermions' particles (electron) $\left(x_{0}\right)$ in diexciton system for calculating spin-spin and spin-orbital effects will be so that total vector potential effect resulted from uni-photon exchange $U v_{s}(x)$ have overlap with the potential resulted from electron converge $U_{s}(x)$. Using oscillator representation method and moving to Ha milton sphere coordination, system would be calculated (oscillation representation method and calculations resulted from it are discussed in this article[20]). Doing required replacements, for two potential we would have (we did this calculation for exotic atom with Coulomb potential[21]):

$$
\begin{gathered}
U_{v}\left(x_{0}\right)=\frac{\hbar^{2}}{r_{e}^{*} m_{e}^{*} x_{0}} U_{s}\left(x_{0}\right) \cong \frac{1}{4}\left(\frac{\alpha \beta \hbar}{x_{0} \sqrt{m_{e}^{*}}}\right)^{2}-\frac{\hbar^{2}}{r_{e}^{*} m_{e}^{*} x_{0}} \\
\omega=\frac{\alpha \beta \hbar}{x_{0} \sqrt{m_{e}^{*}}}, \quad x_{0}=\frac{\beta \kappa \hbar^{2}}{4 z e^{2} m_{e}^{*}}=\frac{\beta r_{e}^{*}}{4 z}, \\
\xi=\frac{m_{e}}{m_{e}^{*}}, \quad z=z_{1}+z_{2}, \quad r_{e}^{*}=a_{b} \varepsilon \xi=\frac{\kappa \hbar^{2}}{e^{2} m_{e}^{*}}
\end{gathered}
$$

Where $\omega=\omega_{1}=\omega_{2}$-oscillator frequency of electrons and $r_{e}^{*}-$ is effective radius of system. In 1991, Schoberl-Louch-Gromes[22], found spin-orbital equations, while these equations are in force for quantum points and we will use them here. Suppose $\hat{S}_{+}=\hat{S}_{1}+\hat{S}_{2}$ for sum of two electrons' spin in diexciton, and for spin-orbital and spin-spin equations we will have:

$$
\begin{gathered}
\hat{H}_{L S}=\frac{1}{2 m_{1} m_{2} x_{0}}\left[3 \frac{\partial}{\partial x} U_{v}\left(x_{0}\right)-\frac{\partial}{\partial x} U_{s}\left(x_{0}\right)\right](\hat{L} \hat{S})= \\
\frac{1}{\left(x_{0} m_{e}^{*}\right)^{2}}\left[\frac{\omega^{2}}{4}-\frac{2 \hbar^{2}}{x_{0} r_{e}^{*} m_{e}^{*}}\right](\hat{L} \hat{S})_{+}=\sigma_{L S}(\hat{L} \hat{S})_{+}
\end{gathered}
$$

And

$$
\begin{gathered}
\hat{H}_{S S}=\frac{1}{12 m_{1} m_{2}}\left(\hat{S}_{1} \hat{S}_{2}\right) \Delta U_{v}\left(x_{0}\right)= \\
\frac{\hbar^{4}}{6 r_{e}^{*} x_{0}^{3}\left(m_{e}^{*}\right)^{4}}\left(\hat{S}_{1} \hat{S}_{2}\right)=\sigma_{S S}\left(\hat{S}_{1} \hat{S}_{2}\right)
\end{gathered}
$$

Having little reform and replacing spin-orbit and spin-spin Hamilton constant coefficient, the system would be:

$$
\begin{aligned}
\sigma_{L S} & =\frac{\omega^{4}}{4 \hbar^{2} \alpha^{4} \beta^{3} m_{e}^{*}}\left(\alpha^{2} \beta z-2\right) \\
\sigma_{S S} & =\frac{\hbar \omega^{3}}{6 r_{e}^{*}(\alpha \beta)^{3}\left(m_{e}^{*}\right)^{\frac{5}{2}}}
\end{aligned}
$$

Using oscillating method in spherical coordination and changing parameters in equation (5), parameters related to particles' distances in diexciton system for electrostatic coulomb potential ruling in quantumdots and overlap of spin potential would be calculated[23], and we have:

$$
x_{0}=\frac{\beta r_{e}^{*}}{4 z}, x_{3}=\frac{\beta r_{e}^{*}}{8 z}, x_{2}=\frac{\beta r_{e}^{*}}{4 z} \sqrt{\frac{m_{e}^{*}}{m_{h}^{*}}}
$$

Where, $x_{3}$-exciton radius (distance between mass center of two holes and two electrons). $x_{2}$-distance between two holes. $x_{0}$-distance between two electrons. As we know, exciton radius will be calcu lable through separating sections related to mass center moves and relative movement through Wannier Equation[24] that is:

$$
R_{e x}=\frac{\kappa \hbar^{2}}{\mu e^{2}}, \quad \mu_{1}=\frac{m_{e}^{*} m_{h}^{*}}{m_{e}^{*}+m_{h}^{*}}
$$

Where, $R_{e x}$ - Bohr radius of exciton or relative distance between electron and hole, $\mu_{1}$ - constituent mass of exciton system, $m_{e}^{*}, m_{h}^{*}$ - effective mass of electron and hold. Relation of exciton radius resulted from Wannier equation in homogeneous environment with $\varepsilon$ dielectric constant coefficient and exciton radius founded for quantum dots between inhomogeneous environments with the same constant dielectric coefficient $\varepsilon$ in oscillator method is: 


$$
\frac{x_{3}}{R_{e x}}=\frac{\beta m_{h}^{*}}{8 z\left(m_{e}^{*}+m_{h}^{*}\right)}
$$

Now using equation $(7,8)$ and $(12)$, we will determine constant coefficient of spin Hamiltonian and exciton radius:

$$
\sigma_{L S}=\frac{m_{e}^{*} \hbar^{2}\left(\alpha^{2} \beta z-2\right)}{\mu}\left(\frac{4 z}{\beta \mu}\right)^{3} \frac{1}{R_{e x}^{4}}
$$

and

$$
\sigma_{S S}=\frac{4 \hbar}{3 m_{e}^{*} r_{e}^{*}}\left(\frac{2 \hbar z}{\beta \mu}\right)^{3} \frac{1}{R_{e x}^{3}}
$$

Above equations clearly determine that there is a direct relation between spin Hamiltonian constant coefficient with visual load sumnear to layers' border and an indirect relation between system reduced mass and exciton radius.

\section{Conclusions}

In this study we worked on spin effects in Nano-crystals constructed by diexciton system in space between $\left(A_{3} B_{5} \mid A_{x} C_{x-1} B_{5}\right)$ nanostructures. Oscillating calculation method has been used to calculate this effect and results of moving form general coordination to Jacobean coordination have been fully discussed. The method based on the investigation of the asymptotic behavior of the polarization loop function for diexciton in an external electro-magnetic field and oscillator representation method. We determined the spin-orbital interaction Hamiltonian with the relativistic corrections and defined spin-spin and spin-orbital Hamilton constant coefficient.

In addition we have shown that how spin Hamiltonian constant coefficients $\sigma_{L S}, \sigma_{S S}$ of exciton system between inhomogeneous environments are related to exciton radius of homogeneous environment. Diagrams for some of nanostructures show that spin effect increase with increase of visual charges (z), and get to steep slop along decrease in dielectric relative coefficient in potential well $\varepsilon$ and electron distance decreases due to their confinement in quantum dot along with increase of visual charges $(z)$.

\section{Appendix}

The important point of calculations is the definition of Hamiltonian's structure of interaction. Interactions between composite particles are carried out through interchanging of gauge fields, therefore the propagator we shall note in a standard way:

$$
\widetilde{D}\left(\vec{q}^{2}+\frac{s^{2}}{c^{2}}\right) \approx \frac{1}{\vec{q}^{2}+\frac{s^{2}}{c^{2}}}=\int_{0}^{\infty} d x \cdot \exp \left\{-x\left(\vec{q}^{2}+\frac{s^{2}}{c^{2}}\right)\right\}
$$

for a potential of interaction after integration on $d \vec{q}$ we have[25]:

$$
\begin{gathered}
W_{i, j}=\frac{1}{2} \alpha^{2}(-1)^{i+j} \int_{0}^{t} \int_{0}^{t} d \tau_{1} d \tau_{2} \int_{-\infty}^{\infty} \frac{d s}{2 \pi} \int_{0}^{\infty} d x(2 \sqrt{\pi x})^{-3} \times \\
\times \exp \left\{-\frac{\vec{r}^{2}}{4 x}\right\} \sum_{k=0}^{\infty} \sum_{n=0}^{k} \frac{(-1)^{n+k}}{n !(k-n) !} \cdot x^{n} r^{(4)(k-n)}\left(\frac{i s}{c}\right)^{n+k} \times \\
\times \exp \{i s \tau\} \cdot \Xi
\end{gathered}
$$

Where

$$
\begin{aligned}
& \tau=\left(\tau_{1}-\tau_{2}\right) ; \quad \vec{r}=\vec{r}_{i}\left(\tau_{1}\right)-\vec{r}_{j}\left(\tau_{2}\right) ; \\
& r^{(4)}(\tau)=r_{i}^{(4)}\left(\tau_{1}\right)-r_{j}^{(4)}\left(\tau_{2}\right) ; \\
& \Xi=1+\frac{\vec{n}}{c}\left(\dot{\vec{r}}_{i}\left(\tau_{1}\right)+\dot{\vec{r}}_{j}\left(\tau_{2}\right)\right)+\frac{\dot{\vec{r}}_{i}\left(\tau_{1}\right) \dot{\vec{r}}_{j}\left(\tau_{2}\right)}{c^{2}}
\end{aligned}
$$

Here $\tau_{1}$ and $\tau_{2}$ - is considered as propertime particles 1 and 2. We suppose that in initial moment particle rest and interact among themselves only by electric field. In this case we have $\Xi=1$. On the other hand, we consider that $r^{(4)}$ Euclid time is connected with $\tau$-proper time quarks as follows:

$$
r^{(4)}=c\left(\tau_{1}-\tau_{2}\right) \equiv c \tau
$$

Taking into account (3.3) and (3.4), and making out integration on the variables ds, after some simplifications from (3.2) is obtained:

$$
\begin{gathered}
W_{i, j}=\frac{\alpha^{2}}{2}(-1)^{i+j} \cdot \int_{00}^{t} \int_{0}^{t} d \tau_{1} d \tau_{2} \dot{Z}_{\alpha}^{(i)}\left(\tau_{1}\right) \times \\
\times D_{\alpha \beta}\left(Z^{(i)}\left(\tau_{1}\right)-Z^{(j)}\left(\tau_{2}\right)\right) \dot{Z}_{\beta}^{(j)}\left(\tau_{2}\right)= \\
\frac{\alpha^{2}}{8 \pi}(-1)^{i+j} \int_{0}^{t} \int_{0}^{t} d \tau_{1} d \tau_{2} \frac{\delta\left(\tau_{1}-\tau_{2}\right)}{\left|\vec{r}_{i}\left(\tau_{1}\right)-\vec{r}_{j}\left(\tau_{2}\right)\right|}+\frac{\alpha^{2}}{8 \pi} \times \\
\times(-1)^{i+j} \sum_{k=1}^{\infty} \frac{(-1)^{k}}{(2 k) ! c^{2 k}} \int_{0}^{t} d \tau \frac{\partial^{2 k}}{\partial \tau^{2 k}}\left(\left|\vec{r}_{i}\left(\tau_{1}\right)-\vec{r}_{j}\left(\tau_{2}\right)\right|^{2 k-1}\right) \equiv \\
W_{i, j}^{(1)}+W_{i, j}^{(2)}
\end{gathered}
$$

Where

$$
\begin{gathered}
Z_{\alpha}(\xi)=(x-y)_{\alpha} \xi+y_{\alpha}-2 \sqrt{s} B_{\alpha}(\xi) \\
\vec{r}(\tau)=\vec{r}_{1}\left(\tau_{1}\right)-\vec{r}_{2}\left(\tau_{2}\right) \\
W_{i, j}^{(1)}+W_{i, j}^{(2)}= \\
-W_{1,1}^{(1)}+2 W_{1,2}^{(12)}-W_{2,2}^{(1)}=\int_{0}^{t} d \tau\left(-\frac{\alpha_{s}}{r(\tau)}+V(0)\right)
\end{gathered}
$$

\section{REFERENCES}

[1] C. Delerue, M. Lannoo. Nanostructures: Theory and Modelling. ISBN-10: 3540206949, Springer, 2004

[2] R. Buczko, G. Duscher, S. J. Penny cook, and S. T. Pantelides, "Excitonic Effects in Core-Excitation Spectra of Semiconductors", Phys. Rev. Lett., vol.85(10), p. 2168, 2000

[3] Dineykhan M., Efimov G.V., Oscillator representation method, Int. J. Mod. Phys. A, vol.4, pp. 4977-4983, 1989

[4] M. Dineykhan, G. V. Efimov, Kh. Narmsrai, Investigation of Green Functions and the Parisi-Wu Quantization Method in 
Background Stochastic Fields, Fortsh. Phys. Vol.39, p. 259, 1991

[5] R. P. Feynman, A. P. Hibbs, Quantum Mechanics and Path Integrals, Ed. New York McGraw-Hill, 1965

[6] M. Dineykhan, A. Jahanshir, S. K. Sakhyev, Definition of masses of bound states, J. Poisk, vol.3, p.199, 2003

[7] M. Dineykhan, A. Jahanshir, D. S. Ashimov, S. Sakhyev, On the structure of QCD string, proceeding of 2th Eurasian Conference on "Nuclear Scence and ITS Application" Almaty, Kazakstan, p.114, 2002

[8] N. Fröman, O. Fröman, Physical problems solved by the phase-integral method, Cambridge University Press, ISBN: 0521812097, 2002

[9] L.D. Landau, E.M. Lifshitz. Quantum Mechanics: Non-Relativistic Theory. Vol. 3 (3rd ed.). Pergamon Press. ISBN 978-0-080-20940-1., p. 506-520, 1977

[10] L. Bergmann, C. Schaefer, and W. Raith, Constituents of Matter: Atoms, Molecules, Nuclei and Particles Berlin: Walter de Gruyter, , ISBN 3110139901 ,p.20-40, 1997

[11] M. Dineykhan, G.V.Efimov, On Bound States in the Quantum Field Theory, The First International Sakharov Conference on Physics was held on May 27-31, in Moscow, USSR, 1991

[12] M. Dineykhan, G.V. Efimov, Ganbolcl G. and Neclelko S.N., Oscillator representation in quantum physics, Lecture Notes in Physics, Springer- Verlag, Berlin, vol. 26, 1995

[13] M.Dineykhan, R. G. Nazmitdinov, On analytical solutions to the problem of the Coulomb and confining potentials, Yad. Fiz., vol.62, p. 143, 1999
[14] M. Dineykhan, G.V. Efimov, The Schrodinger equation for the BoundState Systems in the Oscillator Representation, Reports on Mathematical Physics, vol.36, p. 287, 1995

[15] M. Dineykhan, G.V. Efimov, Zeeman Effect in the oscillator representaion, Yad. Fiz. vol.59, p.862, 1996

[16] M.A. Semina, V. Sergee, R.A Suris, Semiconductors and Semimetals Phys. and Tech Semic.,vol.42, p. 1459, 2008

[17] G. Dresselhaus, Effective mass approximation for excitons, J.. phys. Chem. Solids., vol.1(1/2), p. 15, 1956

[18] M. Dineykhan, at al., Effective Hamiltonian in Scalar Electrodynamics, Booklet, P2-2002-197, Joint Institute for Nuclear Research, Dubna, Russia. 2002

[19] N.A. Gipius, V.D. Kulakovsky, Effect of electric field redistribution on the electronic and optical properties of nanostructures, UFN, vol.167, p.558, 1997

[20] A. Jahanshir, Spin in a quantum dot, proceedings of 1st National Conference on "Nano Science and Technology", Pay amenour University, Yazd, Iran, p.507-511, 2011

[21] W. Lucha, F. Schoberl, D. Gromes, Bound states of quarks Phys. Rep., 200, 1991, 127-135

[22] K. Mackowski, at. al., Sensitivity of exciton spin relaxation in quantum dots to confining potential, App.Phys. Lett., vol. 86(10), 2005

[23] E. Tsitsishvili, Mechanisms of exciton spin relaxation in quantum dots, physica status solidi (b) basic solid state physics, vol.10(5), p.1552, 2006

[24] M. Dineykhan, at. al., On the structure of QCD string, proceeding of the 2 th Eurasian Conference on "Nuclear Science and ITS Applications", Almaty, Kazakhstan, p. 144, 2002 